\title{
Magnetic Remanence of Stainless Steel and Titanium Alloy Orthopaedic Implants
}

\author{
Norhasiza Mat Jusoh ${ }^{a, *}$, Arif Faddilah Mohd Noor ${ }^{b}$, Suffian Mohamad Tajudin ${ }^{b}$, \\ Mohd Hadizie Din ${ }^{\mathrm{c}}$, Mohd Ezane Aziz ${ }^{\mathrm{d}}$, Wan Ahmad Kamile
}

${ }^{a}$ Faculty of Medicine, Universiti Sultan Zainal Abidin, Malaysia, Kuala Nerus, 21300, Malaysia; ${ }^{\text {b }}$ School of Medical Imaging, Faculty of Health Sciences, Universiti Sultan Zainal Abidin, Kuala Nerus, 21300, Malaysia; ${ }^{c}$ Department of Orthopedic, School of Medical Sciences, Universiti Sains Malaysia, Kota Bharu, 16150, Malaysia; ${ }^{d}$

Department of Radiology, School of Medical Sciences, Universiti Sains Malaysia, Kota Bharu, 16150, Malaysia; ${ }^{e}$ Formerly at Department of Radiology, School of Medical Sciences, Universiti Sains Malaysia, Kota Bharu, 16150, Malaysia

*For correspondence: norhasiza@gmail.com

Received: 19 January 2021 Accepted: 15 October 2021

(C) Copyright Jusoh. This article is distributed under the terms of the Creative Commons Attribution License, which permits unrestricted use and redistribution provided that the original author and source are credited.
Abstract Stainless steel and titanium alloys are common materials for orthopaedic implants. However there is a lack of information and studies on magnetic remanence of implants used in clinical practice. The aims of this study are to investigate the composition and the presence of magnetic remanence for these two orthopaedic implant materials. These two factors may cause implant instability and heat problems as well as degradation of the images quality if the patients undergo magnetic resonance imaging (MRI) examination. The magnetic hysteresis loop and remanence status of stainless steel and titanium alloy orthopaedic implants were investigated with a vibrating sample magnetometer (VSM). Both samples of stainless steel and titanium alloy had been exposed to external magnetic fields up to $1 \mathrm{~T}(10000 \mathrm{G})$ and $1.4 \mathrm{~T}(14000 \mathrm{G})$, respectively. The compositions of these two orthopaedic implant materials were studied using a scanning electron microscope with energy dispersive X-ray analysis (SEM-EDX). The results of the study demonstrated that ferrous and nickel compositions in stainless steel alloy orthopaedic implants contributed to the residual magnetism, as shown in the hysteresis loop. The titanium alloy orthopaedic implant sample does not contain any ferromagnetic elements. After exposure to a magnetic field, the stainless steel values of retentivity, coercivity and magnetisation are significantly higher compared to those of the titanium alloy. The stainless steel orthopaedic implant sample demonstrates a typical hysteresis loop that suggests the existence of magnetic remanence. In contrast, the titanium alloy orthopaedic implant sample showed no significant remanence phenomenon. By considering the existence of magnetic remanence in the implant is important as potential effect on the MRI image quality.

Keywords: magnetic hysteresis, magnetic remanence, stainless steel, titanium alloy, orthopaedic implants.

\section{Introduction}

Orthopaedic implants are made from a range of materials which can be categorized as ferromagnetic metals (stainless steel and cobalt chromium (CoCr) alloys), non-ferromagnetic metals (titanium alloy) or non-metals (ceramics, polyethylene). Metal and alloys have been used for orthopaedic implants for decades. This article focuses on magnetic remanence, one of the magnetic properties of the orthopaedic implant materials that are underreported. Two commonly used metals for orthopaedic implants are 
surgical grade stainless steel and titanium alloys. Magnetic remanence or residual magnetism after an extrinsic magnetic field was removed were investigated.

The majority of medical grade stainless steel is an alloy called 316L. Stainless steel was utilised in the past and continues to be the preferred material for a wide range of orthopaedic implants. It is commonly used in the manufacturing of orthopaedic screws, plates, sliding hip screws, some flexible nails, cerclage cables, early generation rigid intramedullary nails and non-permanent implants, such as internal fixation devices [1,2]. Clinically relevant mechanical properties of stainless steel include corrosion resistance, poor fatigue strength and liability to sustain plastic deformation. Titanium is a common material in a variety of orthopaedic implants, including total hip femoral stem, total shoulder arthroplasty components, intramedullary rods and spine surgery implants [3,4]. Titanium alloy is widely use in orthopaedic implants because of the advantages of the retained and predictable mechanics of the material, high biocompatible, and long-term stability $[4,5]$.

Over the last few years, the number of patients with orthopaedic implants has increased substantially [6]. Metal artefacts are common in medical imaging and very common in computed tomographic (CT) and magnetic resonance imaging (MRI). Artefacts arising from metallic orthopaedic implants can degrade CT and MRI images, restricting their clinical diagnostic usage. One study reported that metal artefacts occurred in $18 \%$ of MRI examinations [7]. Both ferromagnetic and non-ferromagnetic materials induce artefacts [8]. Beam hardening is one of the significant artefacts in CT images due to the polychromatic nature of X-ray energy. As the beam passes through an object, it becomes "harder," which means energy increases, because the lower energy photons are absorbed more rapidly than the higher-energy photons. The mismatch created by this large variation in the attenuation coefficient causes CT scans of patients with metal implants to manifest streaking artefacts on an image that result in obscuration of anatomical information [9-11]. Metal artefacts in CT images are not only caused by beam hardening. Other physical effects, such as scattered radiation, noise and nonlinear partial volume, also result in CT image artefacts [12]. MRI metal artefacts have four main effects: signal loss, geometric distortion, signal pile-up and failure of fat suppression. All these effects are worse with higher field strength, and with ferromagnetic alloys rather than titanium [13,14]. Artefacts on MRI images obtained in patients with metallic implants are also produced by the large differences between the magnetic properties of human soft tissues and those of the implanted metals. When an object is placed within a homogeneous magnet, the object produces inhomogeneities in the local magnetic field that interrupt the imaging gradient field. The resultant imaging distortions are called magnetic susceptibility effects [13,15-19]. Despite increasing discussion of CT and MRI artefacts, a dearth of reports on the magnetic remanence aspect of orthopaedic metallic materials could be found. To achieve better understanding of orthopaedic implants causing MRI artefacts, this study will take one step backward to investigate the magnetic remanence of two commonly used metallic orthopaedic materials.

Remanence or residual magnetism is the magnetic flux that is left behind in a substance after an external magnetic field is removed. Magnetic hysteresis exists when an external magnetic field is applied to ferromagnetic material in one direction and the atomic dipoles align themselves with it. Even when the magnetic field is withdrawn, part of the alignment are maintained, and the material has become magnetised. It must be driven back to zero or demagnetised by a magnetic field in the reverse direction. If an alternating magnetic field is applied to the material, such as changing the magnetic field and gradient during MRI scan averaging, echo-planar imaging, frequency encoding and phase encoding, its magnetization will create a hysteresis loop. The detectability of the magnetisation curve is the property called hysteresis and it is related to the presence of magnetic domains in the material. Once the magnetic domains are reoriented, it requires some energy to return them to the original state. The term retentivity is used for magnetic remanence measured in units of magnetic flux density [20-22]. Remanence $\mathrm{Br}$ is a measurement for the magnetic flux density that remains in the sample after an external magnet field is removed. T (Tesla) is used as the unit of measurement for magnetic induction and, magnetic flux density. The unit of measurement previously used was the $G$ (Gauss) [23]. $1 \mathrm{~T}$ is equal to 10,000 G. 


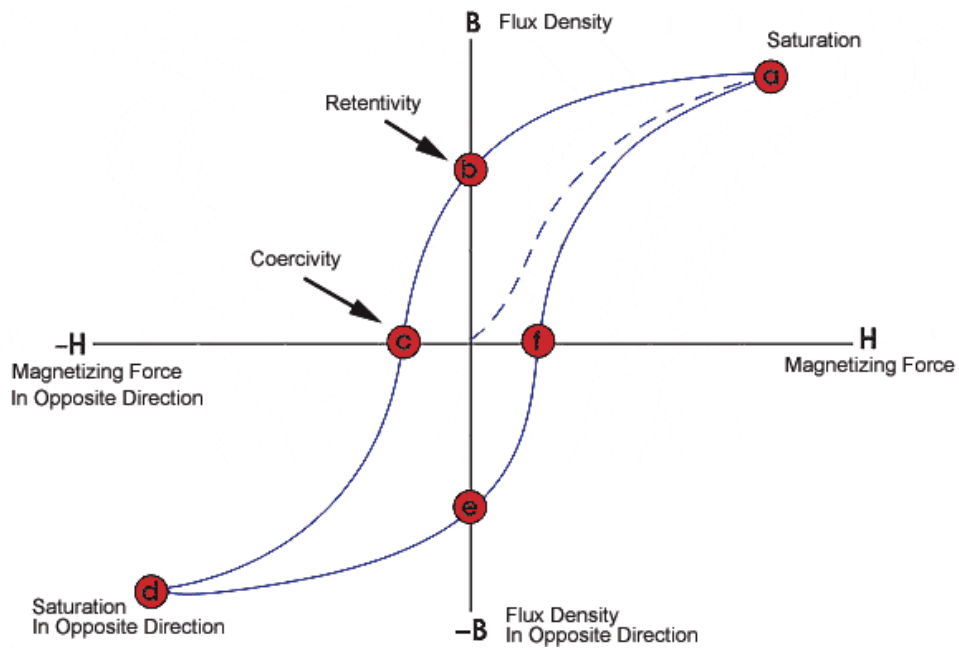

Figure 1. A magnetic hysteresis loop shows the relationship between the induced magnetic flux $B$ and the magnetising force $\mathrm{H}$ (Adapted from https://www.nde-ed.org/Physics/Magnetism/Demagnetization. xhtml)

The hyteresis loop provides the value of rentivity and coercivity of a material is demonstrated in Figure 1. It is produced by measuring the magnetic flux of material while the magnetising force is switched. The material that has never been previously magnetised or has been completely demagnetised will follow the dotted line as $\mathrm{H}$ is increased. As the line exhibits, the larger the amount of current applied $(\mathrm{H}+)$, the stronger the magnetic field in the component $(\mathrm{B}+)$. At point "a" almost all of the magnetic domains are aligned and this is called saturation point. Further increase in the magnetising force will generate very little rise in magnetic flux.It should be noted that saturation magnetisation is an intrinsic property of a material, which does not depend on the shape or size of the material [24]. When $\mathrm{H}$ is decreased to zero, the curve will move from point "a" to point "b." At this point, it can be seen that some magnetic flux remains in the material even though the magnetising force is zero. This is referred to as the point of retentivity on the graph and indicates the remanence or level of residual magnetism in the material. Some of the magnetic domains continue aligned but some have lost their alignment. As the magnetizing force is reversed, the curve moves to point "c", where the flux has been decreased to zero. This is called the point of coercivity on the curve. The reversed magnetising force has flipped enough of the domains so that the net flux within the stainless steel is zero. The force required to eliminate the residual magnetism from the material is called the coercive force or coercivity of the material. As the magnetising force is increased in the negative direction, the material again became magnetically saturated but in the opposite direction (point "d"). Decreasing $\mathrm{H}$ to zero brings the curve to point "e." It has a level of residual magnetism equal to that achieved in the other direction. Increasing $\mathrm{H}$ back in the positive direction will return $\mathrm{B}$ to zero. The curve did not go back to the origin of the graph because some force is required to eliminate the residual magnetism. The curve take a different path from point " $\mathrm{f}$ " back to the saturation point where it with complete the loop [25].

In this study, a scanning electron microscope (SEM-EDX) was used to study the detailed composition of stainless steel and titanium alloy orthopaedic implant samples. Then a vibrating sample magnetometer (VSM) was used to measures the magnetic properties. The objectives of this study are to determine the magnetic hysteresis loop and remanence status of two common orthopaedic implant materials, stainless steel and titanium alloy, using VSM. Detailed composition and remanence status of orthopaedic implants will provide more information on the magnetic material properties of orthopaedic implants. This will assist in the selection decision of the type of materials to use, particularly if the patients require MRI examinations after surgery. 


\section{Materials and methods}

\section{Samples}

Two samples of orthopaedic implant materials which are commonly used in the Orthopaedic Department, Hospital University Sains Malaysia, titanium alloy and stainless steel, were selected for this study. Among all titanium alloy and stainless steel orthopaedic implants available in the institution, the cannulated screw was chosen because it is small and commonly use in multiple orthopaedic surgical procedures. Orthopaedic screws from titanium alloy and stainless steel were used in this study as shown in Figure 2 (a) and (b), respectively. Each cannulated screw was cut to approximately $4 \mathrm{~mm} \times 4 \mathrm{~mm} \times 3 \mathrm{~mm}$ (length $\mathrm{x}$ width $\mathrm{x}$ thickness) in size as in Figure 2(c). The stainless steel and titanium alloy samples were weighted $0.34 \mathrm{~g}$ and $0.19 \mathrm{~g}$ respectively. Each sample was then analysed with VSM and SEM-EDX at room temperature.

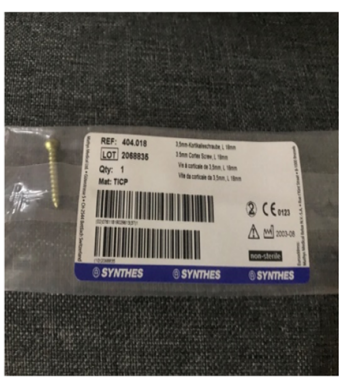

(a)

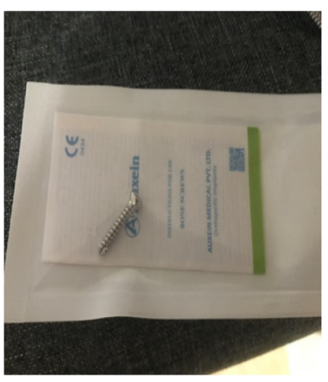

(b)

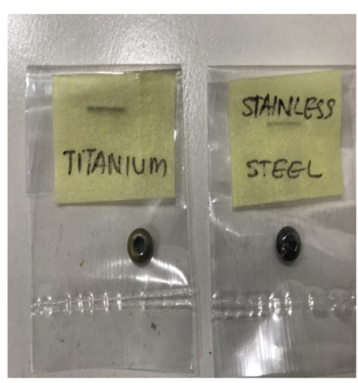

(c)

Figure 2. (a) and (b) Titanium alloy and stainless steel orthopaedic screw. (c) A small piece of each sample analyse using SEM-EDX and VSM.

\section{Elemental composition analysis}

A FEI ESEM Quanta 450 model with an EDX spectrometer was used for elemental composition analysis. SEM-EDX imaging was performed in high vacuum mode as the samples are metallic. Both samples were bombarded with an electron beam at 15 kilovolts $(\mathrm{kV})$. The working distance between the sample to the SEM lens was set at $10 \mathrm{~mm}$.

\section{Magnetic remanence experiment}

The experiment utilizing VSM to determine the remanence status of stainless steel and titanium alloy orthopaedic implant materials was carried out. The system recorded magnetization curves at the room temperature within the magnetic field range of 0 to $1.4 \mathrm{~T}$. Time constant setting for each measurement point was set at 10 second/point. One loop analysis was conducted. The instrument used was a 7404-S Lake Shore, Cryotronics with maximum 1.5 T magnetic field source. The system application software was IDEAS VSM for system operation, data acquisition, and analysis. Both samples were exposed to a magnetic force until saturation magnetic flux was reached. This indicated by after the saturation point, any additional external magnetic field will produce very little increase in magnetic flux . Therefore after saturation, the magnetic force exposure was terminated. Then, the magnetic force was reversed and increased in the negative direction until saturation was reached. The magnetic force was then re-exposed in the positive direction until saturation was reached again. The measurement was conducted one time on each sample. The maximum uncertainty for the setting 10 second/point to be $100 \mathrm{nemu}\left(0.1 \times 10^{-6}\right.$ emu). The data were analysed and plotted into graphs using the IDEAS VSM software, and the hysteresis loop was generated by measuring the magnetic flux of a ferromagnetic material while the magnetising force was changed. 
Jae-Woong et al reported 410 Martensitic Stainless steel measures magnetic hysteresis loops by using VSM before and after proton irradiation, the saturation magnetic field was measured $0.1 \mathrm{~T}$ [26]. Previous study also reported the magnetisation curves of the Ti-6Al-4V specimens before and after exposure, at high magnetic field intensity $0.9 \mathrm{~T}$ (more than $7 \times 10^{5} \mathrm{~A} / \mathrm{m}$ ) almost become straight lines [27].

\section{Results and discussion}

\section{Elemental Composition of Stainless-Steel and Titanium Alloy Orthopaedic Implants}

The two samples of stainless steel and titanium alloy cannulated screw orthopaedic implants were analysed by SEM (model FEI ESEM Quanta 450) with an EDX spectrometer for elemental composition analysis, as shown in Figures 3 and 4, respectively. Both SEM images of the samples shown within the figures were obtained at 500X magnification levels. The peaks from 1 till $9 \mathrm{keV}$ in both spectra are due to the emission of the K-edge characteristic X-ray as a result of the incident electron beam energy on the sample. It must be noted that the emitted energy of the X-rays is highly specific to individual elements. Therefore, the elements within the sample could be identified by the EDX method.

The weight fraction of each element in the stainless steel and titanium alloy samples are presented in Table 1. The stainless steel alloy sample consists of ${ }^{26} \mathrm{Fe},{ }^{24} \mathrm{Cr},{ }^{28} \mathrm{Ni},{ }^{6} \mathrm{C}$ and ${ }^{8} \mathrm{O}$. The main elements which are primarily used as an alloy element, such as iron $\left({ }^{26} \mathrm{Fe}\right)$, chromium $\left({ }^{24} \mathrm{Cr}\right)$ and nickel $\left({ }^{28} \mathrm{Ni}\right)$ appear in the stainless steel sample $[28,29]$. Beside iron, nickel is known to have magnetic properties [30,31]. Chromium has unique magnetic properties because it is the only solid element which demonstrates antiferromagnetic effects at and below room temperature. Above $38{ }^{\circ} \mathrm{C}$, chromium changes its magnetic properties to paramagnetic [32].

The titanium alloy sample consists of three main elements, ${ }^{6} \mathrm{C}(4.35 \%),{ }^{8} \mathrm{O}(28.15 \%)$ and ${ }^{22} \mathrm{Ti}(67.50 \%)$. The sample has no elements with magnetic properties, so it will not be affected by an external magnetic field. However, if the titanium alloy has ${ }^{26} \mathrm{Fe}$, it may exhibit a hysteresis loop [33]. Neither samples contains aluminium. Current orthopaedic implants are developed with no aluminium or vanadium, which are known to be toxic to human soft tissues [34,35].

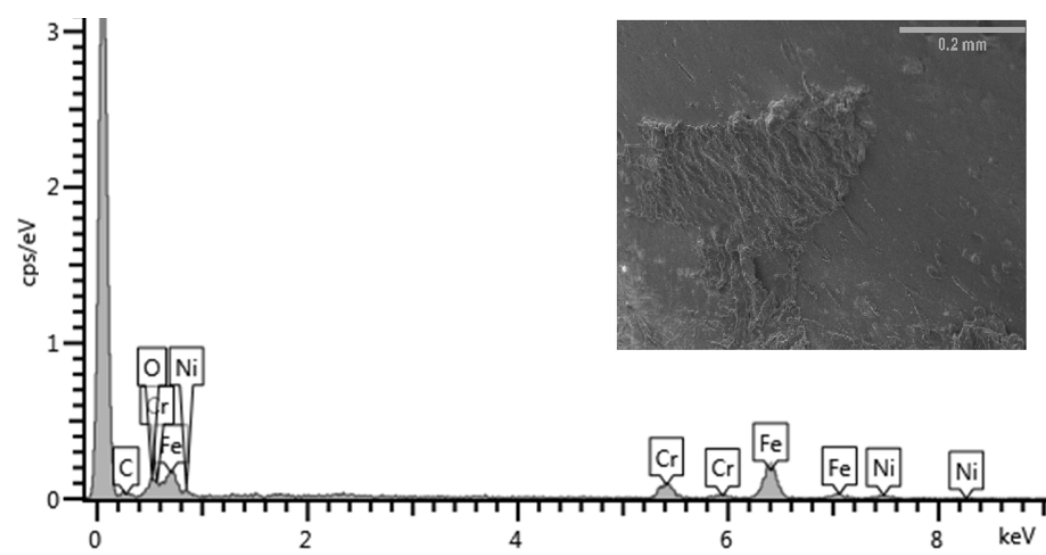

Figure 3. Elemental analysis from an EDX spectrum of a stainless-steel sample. The inset is an SEM image of the sample. 


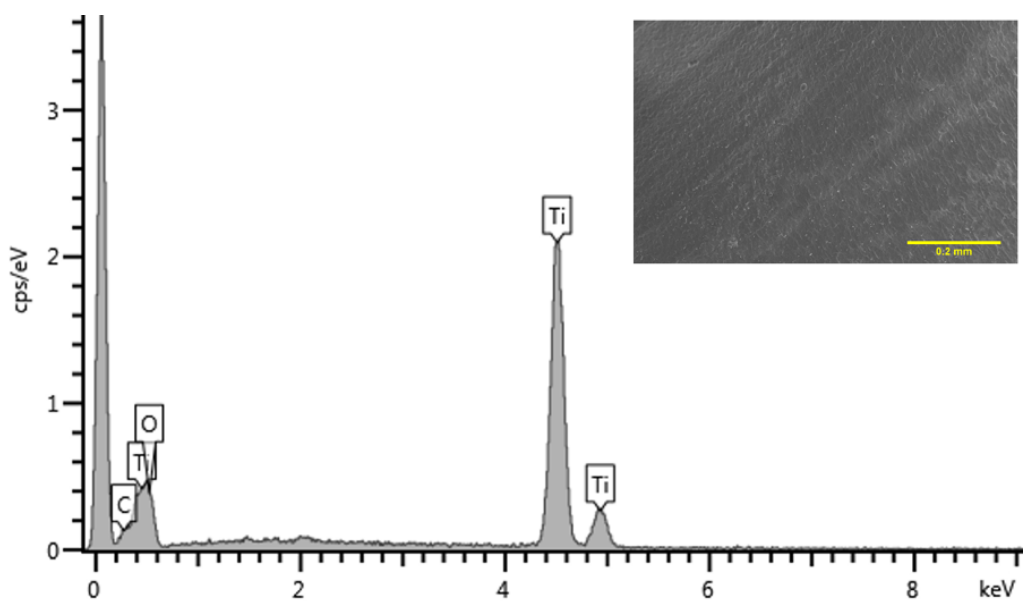

Figure 4. Elemental analysis from an EDX spectrum of a titanium sample. The inset is an SEM image of the sample.

Table 1. Weight percentages of elements in stainless-steel and titanium samples

\begin{tabular}{ccc}
\hline \multirow{2}{*}{ Element } & \multicolumn{2}{c}{ Weight Fraction (\%) } \\
& Stainless Steel & Titanium \\
\hline${ }^{6} \mathrm{C}$ & 6.87 & 4.35 \\
${ }^{8} \mathrm{O}$ & 6.87 & 28.15 \\
${ }^{22} \mathrm{Ti}$ & 0 & 67.50 \\
${ }^{24} \mathrm{Cr}$ & 15.60 & 0 \\
${ }^{26} \mathrm{Fe}$ & 62.29 & 0 \\
${ }^{28} \mathrm{Ni}$ & 8.37 & 0 \\
\hline
\end{tabular}

\section{Remanence Phenomenon (Hysteresis loop)}

The hysteresis loops from the two samples were obtained using the VSM technique, which is used widely for ferromagnetic materials to examine the magnetic properties of various materials when exposed to a strong external magnetic field. Differentiation of the hysteresis curves and the size of the area inside the hysteresis loop give information on magnetic remanence.

In the measurements, both samples were exposed to a strong magnetic field up to 1.4 Tesla (T), which induces magnetisation in the sample. The loop is generated by measuring the magnetic flux of a material while the magnetizing force is changed. The hysteresis curve for the stainless-steel and titanium samples are shown in Figure 5 (a) and (b), respectively.

The form of this loop informs the properties of the material. The area inside the hystereseis loop shows the energy loss due to the change of the magnetization. The energy is converted into heat. The parameters of saturation, rententivity and coercivity indicate important magnetization properties of material. Rententivity and coercivity indicate how easily the material can be magnetized or demagnetized. Saturation shows the amount of magnetization it can store. In Figure 5, the hysteresis and saturation from both samples are shown. The magnetic remanence, based on the size of the area within the hysteresis curves, is significantly different between the samples. The stainless steel sample (a) is characterized by a high remanence, indicating that it will remain magnetized even after an external 
magnetic field is removed. When an external magnetic field is applied to stainless steel in one direction result in the atomic dipoles align themselves with it. Even when the magnetic field is withdrawn, part of the alignment are maintained, and the material has become magnetised. While the titanium alloy (b) shows a consistent decrease of the magnetic flux with a reversal process.

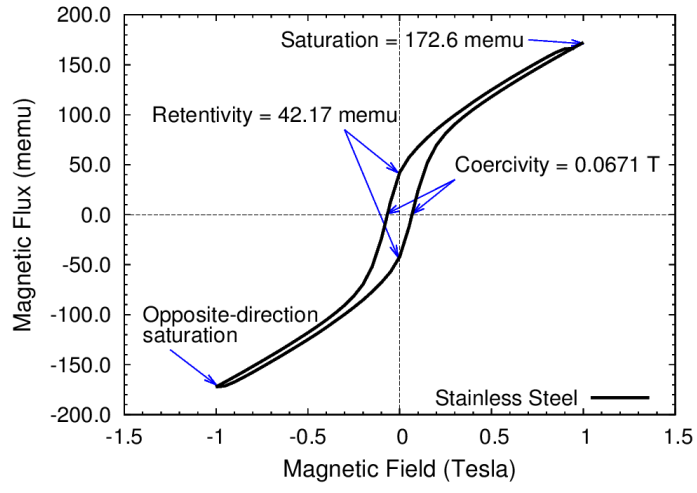

(a)

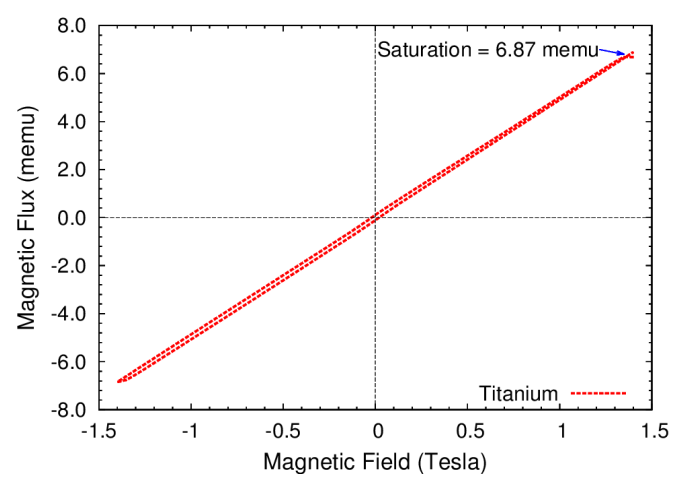

(b)

Figure 5. Hysteresis loop for the stainless steel (a) and titanium alloy (b) samples.

The result is correspond to the ferromagnetic elements composition within stainless steel which are ${ }^{26} \mathrm{Fe}$ and ${ }^{28} \mathrm{Ni}$ (Table 1). Hysteresis loop of stainless steel sample is wide indicate that it retain magnetism and is difficult to demagnetize. It also has high coercivity and therefore a wide hysteresis loop. Titanium alloy sample has low coercivity and therefore hysteresis loop was not able to be plotted in this study. This indicate titanium alloy is easy to magnetise and demagnetize. Stainless steel magnetic hysteresis loop indicate this material can be easily magnetised and at $1.0 \mathrm{~T}$ the magnetisation no further significant increase in magnetisation occur. This called as saturation magnetisation. Gradual reduction of applied external magnetic field, the magnetisation of the stainless steel does not decrease by its original path, but at a slower rate [24].

Table 2. Comparison of measured data of hysteresis loops for stainless steel and titanium alloy

\begin{tabular}{ccc}
\hline Measured Data & Stainless Steel & Titanium Alloy \\
\hline Retentivity & $42.17 \times 10^{-3} \mathrm{emu}$ & $0.11 \times 10^{-3} \mathrm{emu}$ \\
Coercivity & $0.07 \mathrm{~T}$ & $0.02 \mathrm{~T}$ \\
Saturation magnetisation & $172.60 \times 10^{-3} \mathrm{emu}$ & $6.88 \times 10^{-3} \mathrm{emu}$ \\
Saturation (mass) magnetisation & $507.65 \times 10^{-3} \mathrm{emu} / \mathrm{g}$ & $36.21 \times 10^{-3} \mathrm{emu} / \mathrm{g}$ \\
Maximum field & $1.00 \mathrm{~T}$ & $1.40 \mathrm{~T}$ \\
Experiment time & $648 \mathrm{sec}$ & $1695 \mathrm{sec}$ \\
\hline
\end{tabular}

Table 2 summarizes the range of main parameters obtained in the hysteresis loop measurement. In the experiment, both samples of stainless steel and titanium alloy had been exposed to external magnetic fields up to $1.00 \mathrm{~T}$ and $1.40 \mathrm{~T}$, respectively. The stainless steel shows a higher value of retentivity by a factor 387 compared to titanium alloy. The value of coercivity for stainless steel is higher by a factor of 3 than titanium alloy. Both measured results indicate that the stainless steel will maintain the residual magnetic field until the magnetising force of $1.00 \mathrm{~T}$ is removed after the saturation point has been reached. The saturation point values of stainless steel and titanium alloy are $172.60 \times 10^{-3}$ emu and 6.88 $\times 10^{-3}$ emu, respectively. It must be noted that the stainless steel reached the saturation point in a shorter time (648 second) than titanium alloy (1695 second) with $1.00 \mathrm{~T}$ and $1.40 \mathrm{~T}$ magnetic fields, respectively. Thus, the application of both materials as orthopaedic implants and their impacts in magnetic resonance image quality could be understood by considering these factors. 
Stainless steel is an iron-based alloy which is a ferromagnetic substance. There are two main types of stainless steel, austenitic and ferritic, which display different atomic arrangements. Ferritic stainless steels are generally magnetic, while austenitic stainless steels are usually not. A ferritic stainless steel owes its magnetism to the high concentration of iron in its fundamental structure. Ferritic stainless steel is typically unmagnetised. However, when exposed to a magnetic field, it will become magnetised and remains magnetised to some degree even though the applied magnetic field is removed [36]. Titanium, being a paramagnetic substance, is very weakly magnetised by an external magnetic field and loses that magnetism when the external magnetic field is removed [37]. In addition, this study demonstrated that titanium is preferred compared to stainless steel in avoiding the possibility of magnetic field artefacts in MRI examinations. This finding is an important supportive evidence in deciding which orthopaedic implant material is suitable, particularly for patients who are planning for MRI.

\section{Conclusions}

${ }^{26} \mathrm{Fe}$ and ${ }^{28} \mathrm{Ni}$ compositions in stainless steel alloy orthopaedic implants contribute to residual magnetism. The titanium orthopaedic implant sample does not contain ferromagnetic elements, as proven by both SEM-EDX and VSM analysis. The magnetic hysteresis curve obtained from the stainless steel orthopaedic implant showed the occurrence of magnetic remanence. All the measurements of retentivity, coercivity and magnetization values of stainless steel are significantly higher compared to titanium alloy. No magnetic remanence was observed in the titanium alloy orthopaedic implant. This finding is an important piece of evidence if the absence of magnetic remanence in an orthopaedic implant material must be considered, particularly for patients who are planning for MRIs in the future. These findings will lead to future studies on the relationship between magnetic remanence and the associated artefact that may be seen in MR images in the vicinity of a stainless steel implant. It is hoped that the present results will be useful in determining the origin of such artefacts. Based on our studies, the potential effects of magnetic remanence to the surrounding human cells and soft tissues from metal orthopaedic implants within the patients' body after undergoing MRI examination have not yet been discussed. This is another potential study on magnetic remanence, particularly in an era of increasing magnetic resonance imaging usage in clinical practice and numbers of patients with orthopaedic implants worldwide.

\section{Data availability}

Readers can access the data underlying the findings of the study by email the corresponding author

\section{Conflicts of interest}

The authors declare that they have no conflict of interest.

\section{Funding statement}

This study was done under the Fundamental Research Grant Scheme, Ministry of Education Malaysia (FRGS/1/2016/SKK06/UNISZA/03/2).

\section{Acknowledgments}

This work was funded by Fundamental Research Grant Scheme, Ministry of Education Malaysia (FRGS/1/2016/SKK06/UNISZA/03/2). Authors gratefully acknowledge Radiological Department of Hospital Universiti Sains Malaysia administration and staff for their help and support. 


\section{References}

[1] Gyaneshwar T, Nitesh R, Sagar T, Pranav K, Rustagi N. Treatment of pediatric femoral shaft fractures by stainless steel and titanium elastic nail system: A randomized comparative trial. Chin. J. Traumatol. 2016 Aug 01;19(4):213-6.

[2] Bostrom MP, Asnis SE, Ernberg JJ, Wright TM, Giddings VL, Berberian WS, Missri AA. Fatigue testing of cerclage stainless steel wire fixation. J Orthop Trauma. 1994 Oct;8(5):422-8.

[3] Chouirfa $\mathrm{H}$, Bouloussa $\mathrm{H}$, Migonney V, Falentin-Daudré C. Review of titanium surface modification techniques and coatings for antibacterial applications. Acta Biomater. 2019 Jan;83:37-54.

[4] Yılmaz E, Gökçe A, Findik F, Gulsoy HO, İyibilgin O. Mechanical properties and electrochemical behavior of porous Ti-Nb biomaterials. J Mech Behav Biomed Mater. 2018 Nov;87:59-67.

[5] Niinomi M. Mechanical Biocompatibilities of Titanium Alloy for Biomedical Applications. Journal of the Mechanical Mechanical Behavior of Biomedical Material. 2008;1(1):30-42.

[6] Kurtz, S., et al. Projections of primary and revision hip and knee arthroplasty in the United States from 2005 to 2030. J Bone Joint Surg Am. 2007; 4:780-5.

[7] Tomas B, Vincentas V, Saulius L, Rymante G, Egle M, Ilona K. Artifacts in magnetic resonance imaging: how it can really affect diagnostic image quality and confuse clinical diagnosis?. Journal Of Vibroengineering. 2018 Mar; 20 (2):1202-13. Doi:10.21595/jve.2018.19756.

[8] Leclet $\mathrm{H}$. Artifacts in magnetic resonance imaging of the spine after surgery with or without implant. European Spine Journal. 1994; 3 (5). 240-5.

[9] White LM, Buckwalter KA. Technical considerations: CT and MRI imaging in the postoperative orthopedic patient. Semin Musculoskelet Radiol. 2002;6:5-17.

[10] Hsieh J. Image artifacts, in CT. In: Goldman LW FJ, editor. Syllabus: A Categorical Course in Diagnostic Radiology Physics: CT and US Cross-Sectional Imaging. Oak Brook, IL: Radiological Society of North America. 2000;97-115.

[11] Van de Casteele E, Van Dyck D, Sijbers J, \& Raman E. An energy-based beam hardening model in tomography. Physics in Medicine and Biology. 2002;47(23): 4181-4190.

[12] Meyer E, Raupach R, Lell M, Schmidt B, Kachelrie M. Normalized metal artifact reduction in computed tomography. Med. Phys. 2010;37.5482-5493. Doi:10.1118/1.3484090.

[13] Koch KM, Hargreaves BA, Pauly KB, Chen W, Gold GE, King KF. Magnetic resonance imaging near metal implants. J Magn Reson Imaging. 2010;32(4):773-787.

[14] Hargreaves BA, Worters PW, Pauly KB, Pauly JM, Koch KM, Gold GE. Metal-induced artifacts in MRI. AJR Am J Roentgenol. 2011;197(3): 547-555.

[15] Schenck JF. The role of magnetic susceptibility in magnetic resonance imaging: MRI magnetic compatibility of the first and second kinds. Med Phys 1996; 23:815-850.

[16] Sharief M, Aboelmagd Paul N, Malcolm Andoni PT. Magnetic resonance imaging of metal artifact reduction sequences in the assessment of metal-on-metal hip prostheses. Reports in Medical Imaging 2014;7 65-74.

[17] Guermazi A, Miaux Y, Zaim S, Peterfy CG, White D, Genant HK. Metallic artifacts in MR imaging: effects of main field orientation and strength. Clin Radiol 2003;58:322-328.

[18] Eggers G, Rieker M, Kress B, Fiebach J, Dickhaus H, Hassfeld S. Artifacts in magnetic resonance imaging caused by dental material. MAGMA. 2005;18:103-111.

[19] Viano AM, Gronemeyer SA, Haliloglu M, Hoffer FA. Improved MR imaging for patients with metallic implants. Magn Reson Imaging 2000;18: 287-295.

[20] Hyperphysics. Hysteriss. http://hyperphysics.phy-astr.gsu.edu/hbase/Solids/hyst.html. Accessed on 17 Jun 2020.

[21] Principles, demagnetization curve and Magnetic Remanence of Ferrite Magnet. https://www.maruwa-g.com/e/products/ceramic/upfiles/magnet_en.pdf. Accessed on 15 Oct 2020.

[22] Remanence. https://www.britannica.com/science/remanence-magnetism. Accessed on 15 Oct 2020.

[23] Remanence. https://en.wikipedia.org/wiki/Remanence. Accessed on 17 Jun 2020.

[24] Elizaveta M, Shaoying H. Advanced in Magnetic Materials. Routledge Handbooks Online. 2017: eBook ISBN: 9781315371576.

[25] The Hysteresis Loop and Magnetic Properties. https://www.nde-ed.org/Physics/Magnetism/ Demagnetization.xhtml. Accessed on 24 October 2020. 
[26] Jae-Woong L, Surabhi S, Soon-Gil Y, Ho Jin R, Byong-Guk P, Yeon-Ho C, Yong-Tae J, and JongRyul J. Study on Proton Radiation Resistance of 410 Martensitic Stainless Steels under $3 \mathrm{MeV}$ Proton Irradiation. Journal of Magnetics. 2016; 21(2), 183-186. DOI: 10.4283/JMAG.2016.21.2.183.

[27] Xu Z, Qian Z, Zhipeng C, Jiluan P. Effects of Magnetic Field on the Residual Stress and Structural Defects of Ti-6Al-4V. Metals. 2020;10:141.

[28] Er Y, Unsaldi E. The production of nickel-chromium-molybdenum alloy with open pore structure as an implant and the investigation of its biocompatibility in vivo. Advances in Materials Science and Engineering. 2013:1-6. Doi:10.1155/2013/568479.

[29] Manam NS, Harun WSW, Shri DNA, Ghani SAC, Kurniawan T, Ismail MH, Ibrahim MHI. Study of corrosion in biocompatible metals for implants: A review. Journal of Alloys and Compounds. 2017;701 (October):698-715.

[30] Yuanzhi C, Dong-Liang P, Dongxing Li, Xiaohua L. Preparation and magnetic properties of nickel nanoparticles via the thermal decomposition of nickel organometallic precursor in alkylamines. Nanotechnology. 2007;18(505703):1-6.

[31] Vempaire D, Miraglia S, Sulpice A, Ortega L, Hlil EK, Fruchart D, Pelletier J. Structure and magnetic properties of nickel nitrate thin film synthesized by plasma-based ion implantation. Journal of Magnetic and Magnetic Materials. 2004;272-276 (6): E843-E844.

[32] Eric F. Spin-density-wave antiferromagnetism in chromium. Review of Modern Physics. 1998;60:209.

[33] Ciesla M, Junak G, Marek A, Fatique Characteristics of Selected Light Metal Alloys. Arch. Metall. Mater. 2016;61(1):271-274.

[34] Jeffery EH, Abreo K, Burgess E, Cannata J, \& Greger JL. Systemic aluminum toxicity: Effects on bone, hematopoietic tissue, and kidney. Journal of Toxicology and Environmental Health - Part A. 1996;48(6):649-666.

[35] Sansone V, Pagani D, Melato $M$. The effects on bone cells of metal ions released from orthopedic implants. A review. Clinical Cases in Mineral and Bone Metabolism. 2013;10(1):34-40. Doi:10.11138/ccmbm/2013.10.1.034.

[36] Thomas D. Why don't magnets work on some stainless steels? 2006. https://www. scientificamerican.com/article/why-dont-magnets-work-on. Accessed 6 Jun 2010.

[37] Wikipedia, the free encyclopedia. Paramagnetism. https://en.wikipedia.org/wiki/Paramagnetism. Accessed on 17 Jun 2020. 